\title{
Surface and Defect Chemistry of Oxide Materials
}

\author{
Ulrich Aschauer*
}

\begin{abstract}
Oxides and oxide-derived materials find ubiquitous applications in many industrial applications. These materials usually contain point defects, the concentration and type depending on the synthesis and operating conditions. Many of the functional properties are determined or strongly affected by the presence of these point defects. Despite steady advances in experimental techniques it is still difficult to unambiguously identify point defects and resulting atomic-scale mechanisms from experiment alone. Atomic-scale computational approaches such as density functional theory (DFT) provide an alternative approach that ideally complements experimental investigations. The resulting fundamental understanding of defect-induced mechanisms combined with the knowledge of how to tailor a point-defect profile to induce new functionality opens exciting new avenues to engineer novel material properties. This account discusses some case studies of defect-induced functionality in the area of oxide electronics and photocatalysis, giving a general flavour of our current understanding of the role of defects in these applications.
\end{abstract}

Keywords: Catalysis $\cdot$ Defects $\cdot$ Density functional theory $\cdot$ Oxide electronics $\cdot$ Oxides

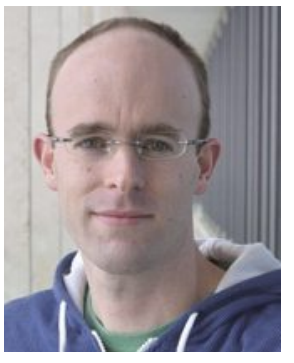

\section{Introduction and Motivation}

Oxides are industrially important materials with ubiquitous applications ranging from refractories over photocatalytic electrodes to resistive switching memories and including many fields in between. ${ }^{[1-3]}$ In these applications, oxides are in many different forms, including polycrystalline ceramics, single-crystalline bulk materials, surfaces, thin films and heterostructures. Often, the principles underlying functionality in a specific application are determined by atomic-scale details in the bulk or at surfaces and interfaces. Despite the development of sophisticated experimental imaging and spectroscopic probes, it still remains difficult to fully understand the atomic-scale structure and resulting mechanisms from experiment alone. Atomicscale computational tools, such as density functional theory (DFT), provide ways to model such atomic arrangements based on quantum-mechanical first principles and are ideally suited to complement experiment in uncovering atomic-scale mechanisms.

Depending on the synthesis conditions, oxides typically contain point defects, the most abundant usually being the oxygen vacancy. While these defects are often seen as detrimental to properties (apart maybe from rendering oxides conductive) it has recently become apparent that they can, in fact, induce new properties, a fundamental understanding of which requires the aforementioned combination of theoretical and experimental investigations. More interestingly though, this raises the question as to how one can engineer point defect-induced properties. Addressing this challenge will require knowledge of both changes in properties in presence of defects as well as the possibility of accurately predicting the synthesis conditions leading to the desired defect profile.

In this account, I will highlight examples of previous work that lead to these questions and describe our general approach to addressing them. The applications I will discuss are within the areas of thin-film oxide electronics and photocatalysis but we have applied this general approach to understanding defect-induced properties also in other areas such as memristive switching or superconductivity. ${ }^{[4,5]}$

\section{Computational Approach}

For our research, we primarily use a method known as density functional theory (DFT) ${ }^{[6,7]}$ which, among quantum mechanics-based techniques, currently offers the best trade-off between accuracy and the size of the systems that one can compute. Compared to conventional quantumchemical methods, DFT does not seek to describe the electronic wavefunction of the collection of electrons but rather assumes an auxiliary system of independent electrons having the same total electron density, while describing their many-body and non-classical (i.e. quantum-mechanical) interaction via a parametrized so-called exchange-correlation functional. This functional is therefore responsible for recovering the real quantum mechanical behaviour and its performance is of crucial importance for the accuracy of the calculation. Since the true universal functional is not known, in practice one has to use approxi- 
mate functionals with varying degrees of complexity. For our work, so-called hybrid functionals (such as HSE06 ${ }^{[8,9]}$ ) currently represent the most accurate yet often still feasible approach. Computationally less demanding gradient corrected functionals are however still routinely used, often combined with suitable corrections such as $\mathrm{DFT}+\mathrm{U}^{[10]}$ to improve their accuracy for transition metal systems of interest in our research. Using these approaches on present-day high-performance computers (HPC), one can routinely perform calculations on transition metal systems of a few hundred atoms.

DFT calculations of the flavour we use are typically carried out under periodic boundary conditions, meaning that the atomic-scale geometry is placed in a simulation cell, which is then repeated infinitely along the three directions of space (see Fig. 1a). The default geometry is thus that of a bulk crystal, other geometries requiring tweaks of the setup. For defect calculation in a bulk material for example, one uses not the crystallographic unit cell but a supercell that is large enough to minimize defect-defect interactions - in other words the defect concentration should be low enough (see Fig. 1b). For surface calculations, we introduce what is known as a vacuum gap, which breaks the periodic linkage along one direction, combined with fixing the bottom part of the resulting 'slab' of material to mimic the presence of a large and rigid bulk (see Fig. 1c). Both the width of the vacuum gap as well as the thickness of the fixed bulk and free surface layers will affect the accuracy of the computed results. For thin-film calculations, one normally neglects surface and hetero-interface effects and considers the effect of epitaxial strain via constraining the in-plane lattice parameters of a suitable bulk cell with one free out-of-plane lattice parameter (see Fig. 1d). When combining these setups and doing for example defects at surfaces, the requirement of a sufficient defect-defect separation is combined with the vacuum-gap setup.

\section{Strain-dependent Bulk Defect Chemistry}

The defect chemistry as a function of the partial pressures (chemical potentials) in the environment is nowadays well estab- (a)

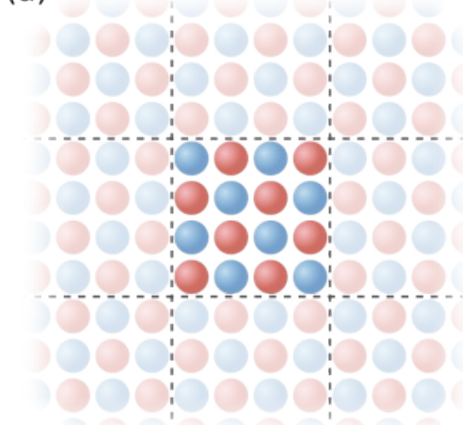

(c)

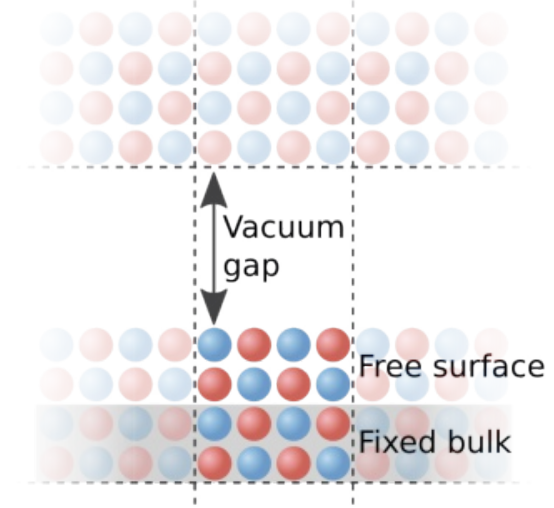

(b)

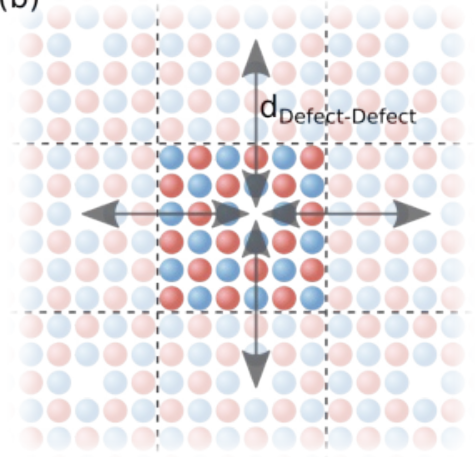

(d)

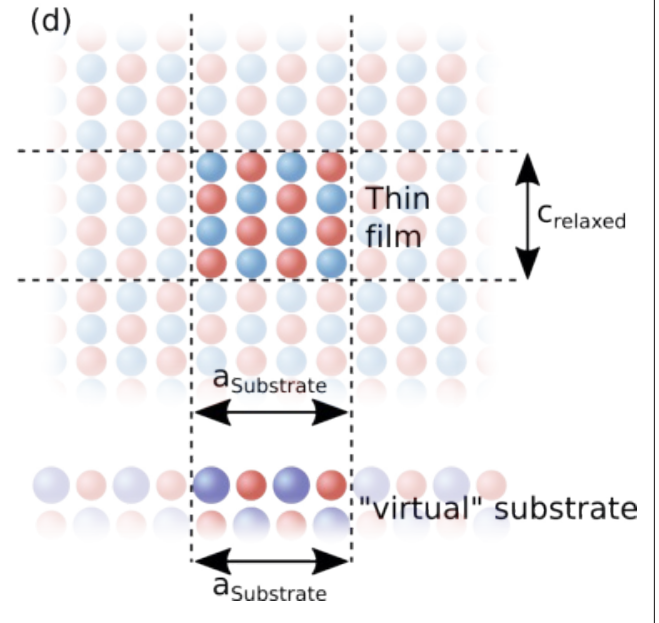

Fig. 1. 2D schematic views of the various computational setups: a) bulk crystal, b) bulk defect, c) surface cell and d) thin film constrained to lattice parameters of a 'virtual' substrate. The dashed lines show the boundaries of the simulation cell. Periodically repeated parts are shown in lighter colors.

lished, ${ }^{[11]}$ the formation energy of a defect being described as

$$
\Delta E_{\text {form }}=E_{\text {defect }}-E_{\text {perfect }}+\sum_{i} n_{i} \mu_{i}
$$

where $E_{\text {defect }}$ and $E_{\text {perfect }}$ are the DFT total energies of the defective and perfect cell respectively, while $n_{i}$ is the number of atoms of type $i$ exchanged with a reservoir, where they have a chemical potential $\mu_{i}$. Experimentally exposing a material to vacuum corresponds to lowering the partial pressure of oxygen (along with that of other gases in the environment). In our setup, this is equivalent to reducing the oxygen chemical potential, which has the effect of a reduced energetic cost $\Delta E_{\text {form }}$ to form oxygen vacancies and results in a higher concentration of these defects.

The relation of defects and volume changes is well-established in the ceramics community, ${ }^{[12]}$ where some defects (formed under oxygen-poor conditions) are known to expand the lattice of an oxide ceramic, while others (formed under oxygen-rich conditions) are known to shrink the lattice. ${ }^{[13]}$ This highlights a direct interdependence of the volume and the defect chemistry of the material, in the above example with the defect chemistry controlling the volume. We can now turn this concept around and postulate that volume changes should affect the defect chemistry. Volume changes could be induced during high-pressure experiments, for example carried out in diamond-anvil cells, but an alternative and technologically more relevant route are epitaxially strained thin films, where the volume changes by constraining two axes of the film to the lattice parameters of the substrate.

In a proof-of-concept study[14] we could show that epitaxial strain indeed has a marked effect on the defect formation energy and hence the defect concentration. Our DFT calculations indicate that the formation energy of an oxygen vacancy in the perovskite oxide $\mathrm{CaMnO}_{3}$ should be reduced by as much as $0.4 \mathrm{eV}$ under $4 \%$ tensile strain as shown in Fig. 2a. This change results from the easier accommodation of the larger $\mathrm{Mn}^{3+}$ ions that form by reduction of the regular lattice $\mathrm{Mn}^{4+}$ to compensate the charge of an oxygen vacancy.

The effect of this seemingly small $(20 \%)$ change in formation energy has huge implications on the defect concentration and the oxygen content of the material as shown in Fig. 2b. Due to the appearance of the formation energy in an Arrhenius exponential coefficient, applying $4 \%$ tensile strain reduces the oxygen content at a typical growth temperature $\left(1000 \mathrm{~K} \cong 730^{\circ} \mathrm{C}\right)$ and ambient oxygen pressure from nearly stoichiometric $(\sim 3)$ to 2.97 , which corresponds to a fairly high vacancy concentra- 


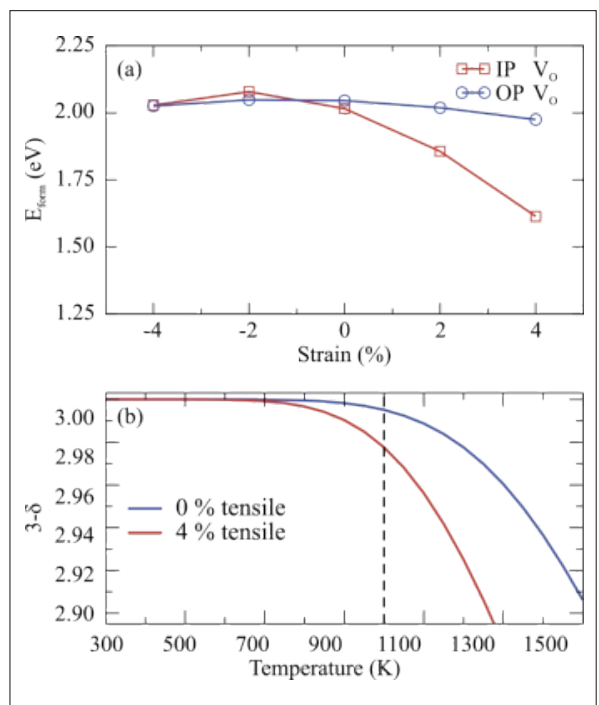

Fig. 2. a) Oxygen vacancy formation energy at ambient conditions as a function of compressive or tensile epitaxial strain for the in-plane (IP) and out-of-plane (OP) oxygen vacancy and b) resulting oxygen content (3- $\delta$ ) as a function of temperature in the unstrained and $4 \%$ tensile strained case. Reprinted (adapted) with permission from ref. [14]. Copyright (2013) American Physical Society.

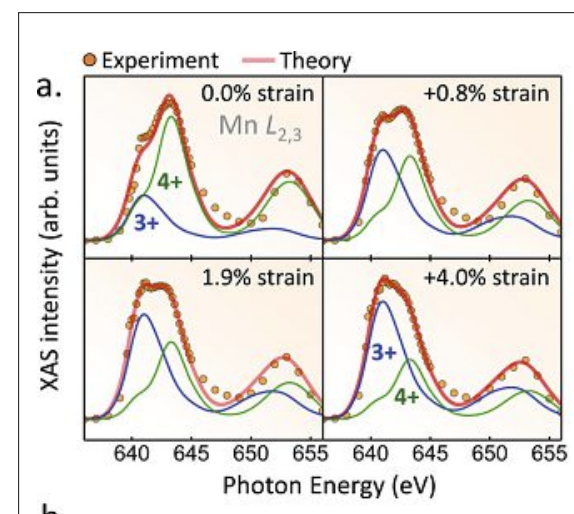

b.

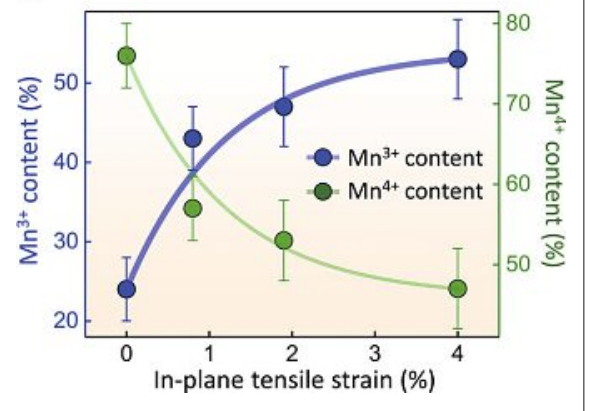

Fig. 3. a) Changes in the strained $\mathrm{CaMnO}_{3}$ $\mathrm{X}$-ray absorption signal and its fit into $\mathrm{Mn}^{3+}$ and $\mathrm{Mn}^{4+}$ components and b) the extracted proportions of respective concentrations, showing at $4 \%$ strain, there are about equal concentrations of $\mathrm{Mn}^{3+}$ and $\mathrm{Mn}^{4+}$ in the film. Reprinted with permission from ref. [15]. Copyright (2017) American Chemical Society.

panied by oxidation. ${ }^{[16]}$ These defects however typically have significantly higher formation energies than anion defects and the effects may thus be smaller than under tensile strain. An experimental analysis of enhanced cation vacancy concentrations is still outstanding.

When an increased oxygen vacancy concentration coincides with other straininduced properties such as ferroelectricity, other fascinating properties result. Oxygen vacancies were for example shown to accumulate at ferroelectric domain walls in tensile-strained $\mathrm{SrMnO}_{3}{ }^{\left[{ }^{[17]}\right.}$ Their presence induces an electrostatic potential barrier that prevents electrons from crossing the vacancy-decorated domain wall (see Fig. $4 a)$, resulting in electrically insulated nano-sized domains in the material as shown in Fig. 4b). These areas can be individually charged, making them of potential interest for example in data-storage applications.

\section{Chemistry of Defective Surfaces}

Defects also have a marked effect on the surface chemistry of materials. On the well-studied $\mathrm{TiO}_{2}$ rutile (110) surface for example, they are known to promote dissociative over molecular adsorption of water. ${ }^{[18]}$ In this situation, the defect is lo-

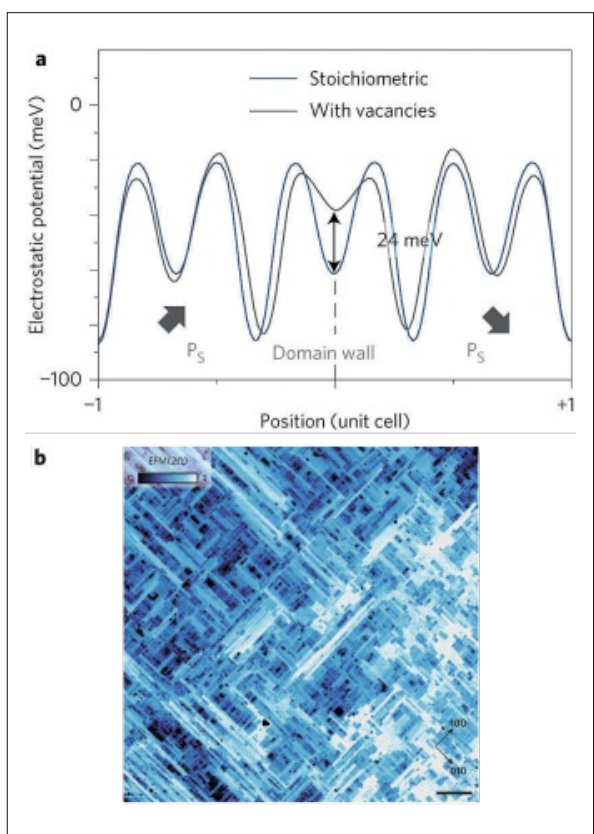

Fig. 4. a) Plane-averaged electrostatic potential across a ferroelectric $90^{\circ}$ head-tail domain wall, showing a $24 \mathrm{meV}$ barrier in presence of oxygen-vacancy decoration of the wall. b) Electrostatic force microscopy image showing conductively separated domains due to the presence of vacancy-decorated domains walls. Reprinted (adapted) with permission from ref. [17]. Copyright (2015) Springer Nature.

cated at the surface, ready to react with the adsorbing molecules. Other surfaces, such as the most stable $\mathrm{TiO}_{2}$ anatase (101) surface do not, however, exhibit surface defects but defects are preferentially located in the subsurface and bulk. ${ }^{[19,20]}$ Somewhat surprisingly, our calculations ${ }^{[21]}$ indicate that such a subsurface vacancy can be extracted to the surface after an $\mathrm{O}_{2}$ molecule adsorbs above the defect (Fig. $5 \AA$ ). $\mathrm{O}_{2}$ molecules are known to act as electron scavengers, taking up excess electrons associated with the vacancy and turning molecular oxygen into a superoxo $\left(\mathrm{O}_{2}^{2-}\right)$ species. This charge transfer destabilizes the local vacancy structure in the subsurface and the vacancy migrates to the surface (Figs 5B and $5 \mathrm{C}$ ), where the superoxo that carries the charge of a regular lattice oxygen ion is incorporated into the vacancy (Figs 5D and $5 \mathrm{E}$ ), forming a so-called bridging dimer $\left(\mathrm{O}_{2}\right)_{0}$ shown in Fig. 5F. This process is nearly barrierless and exothermic by 1.6 eV (Fig. 5G). Following preparation of the surface by electrostatically pulling vacancies from the bulk to the surface by means of a charged scanning tunnelling microscope (STM) tip, this reaction was readily observed in a series of STM images while dosing the surface with $\mathrm{O}_{2}{ }^{[21]}$

We have subsequently shown the role of this bridging dimer species as a reac- shown that compressive strain favours the formation of cation defects that are accom- 
tant in the decomposition of water into terminal $\mathrm{OH}$ groups and as a product in the reduction reaction of $\mathrm{O}_{2}$ in presence of water via formation of intermediate $\mathrm{OOH}$ species. ${ }^{[22]}$ Given that $\mathrm{OH}$ and $\mathrm{OOH}$ species are important intermediates in various oxidation and reduction reactions on oxide surfaces, ${ }^{[23]}$ the bridging dimer is expected to be involved in these reactions on the $\mathrm{TiO}_{2}$ anatase (101) surface. [24]

\section{Alternatives to Oxide Materials}

Oxides such as the aforementioned $\mathrm{TiO}$, have band gaps of the order of $3 \mathrm{eV}$, which is too large to absorb a major portion of the solar spectrum. ${ }^{[2]}$ Despite decades of research, this property renders their economically viable application as photocatalysts questionable. Nitrogen, being less electronegative than oxygen, results in higher energy occupied states at the valence band edge, thus narrowing the band gap. ${ }^{[25]}$ For this reason, oxynitrides, which are mixed anion materials containing both nitrogen and oxygen, are highly promising for these applications. ${ }^{[26]}$ Oxynitrides

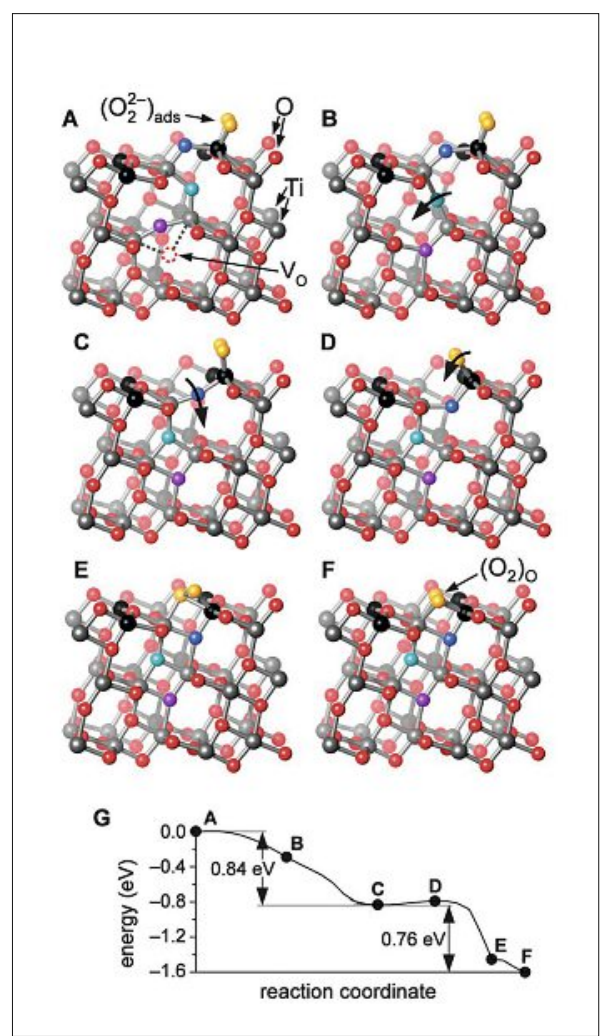

Fig. 5. A)-C) Oxygen vacancy subsurface to surface migration under the effect of an adsorbed $\mathrm{O}_{2}$ molecule, D)-F) followed by the incorporation of the molecule into the vacancy and formation of a $\left(\mathrm{O}_{2}\right)_{0}$ bridging dimer species. G) Energy profile showing the nearly barrierless and strongly exothermic character of the reaction. Reprinted (adapted) with permission from ref. [21]. Copyright (2013), American Association for the Advancement of Science.

of the chemically and structurally flexible perovskite structure further permit tuning of the band gap via cation-induced structural distortions. The defect and surface chemistry of these oxynitride materials is however significantly less established than that of pure oxides.

Over the last two years our work has revolved around establishing surface structures of these computationally challenging materials. While in the bulk oxynitrides typically favour a cis order of the nitrogen anions to maximize the transition metal $d$ anion $p$ overlap, ${ }^{[27,28]}$ polarity compensation at the surface favours a trans order along the surface normal for $\mathrm{LaTiO}_{2} \mathrm{~N}$ (001) (Fig. $6),{ }^{[29]}$ the predicted most stable LaN termination being consistent with experimentally observed La-rich surface layers. ${ }^{[30]}$ In oxynitrides with a 5+ charged B-site such as $\mathrm{SrTaO}_{2} \mathrm{~N}$, we however predict a cis order also at the surface as this anion arrangement already compensates polarity in this case. ${ }^{[31]}$ Additional structural modifications such as the adoption of a layered Ruddlesden-Popper structure can, at least for Ta-based materials, lead to an electronic structure that is highly beneficial for photocatalysis as surfaces states effectively suppress electron-hole recombination. ${ }^{32]}$

Our recent work has also shown that epitaxial strain can be used to tune the anion order and ferroelectricity in $\mathrm{LaTiO}_{2} \mathrm{~N}_{\text {. }}{ }^{[33]}$ As shown in Fig. 7, for compressive strains larger than $4 \%$, LaTiO $_{2} \mathrm{~N}$ adopts a trans nitrogen order and becomes ferroelectric, the two properties being correlated but not

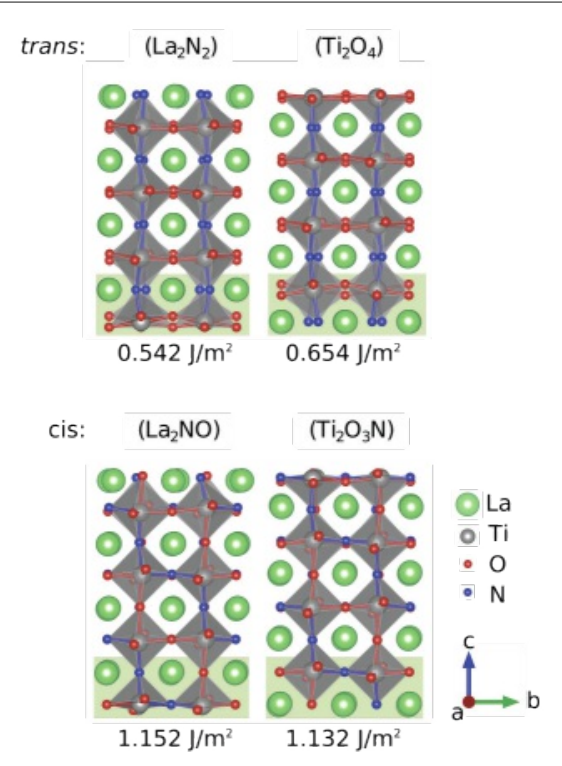

Fig. 6. Anion order at the $\mathrm{LaTiO}_{2} \mathrm{~N}(001)$ surface: trans and cis ordered structures. The trans ordered $\mathrm{La}_{2} \mathrm{~N}_{2}$ termination has the lowest overall surface energy and is expected to be exposed in vacuum. Reprinted (adapted) with permission from ref. [29]. Copyright (2017), Royal Society of Chemistry. causally related.[33] Switching the electrical polarization during the water oxidation cycle could promote adsorption or desorption reactions respectively. This would open an exciting route to engineer catalytic surfaces operating beyond Sabatier's principle, which states that the performance of a catalyst is determined by a competition between adsorption of reactants and desorption of products. ${ }^{[33]}$

\section{Conclusions and Outlook}

Computational methods nowadays effectively complement experimental techniques in understanding defect-induced properties and defect-enabled atomic scale mechanisms. Given our growing understanding of property changes resulting from defects, engineering novel oxide material properties via targeted off-stoichiometry becomes an exciting possibility. Compared to the bulk, surfaces and interfaces offer added complexity as defect formation is altered by the discontinuous crystal structure. The interaction of defects with adsorbing molecules and the exchange of ions across the interface are important areas of research to better understand the thermodynamics and kinetics of defects in these environments and their equilibrium with the bulk. A complete understanding of the defect chemistry in these complex environments is yet to be achieved and is a very active field of research. For some applications (i.e. photocatalysis) anionsubstituted materials such as oxynitrides are more promising than pure oxides. The reduced stability of oxynitrides compared to oxides requires special emphasis on the surface defect chemistry, focussing also on nitrogen defects. Ongoing research shows indeed that for these materials defects are expected to play an active role in photocatalytic reactions, the different behaviour of nitrogen vacancies compared to oxygen vacancies, ${ }^{[34]}$ however, still being an open question.

\section{Acknowledgements}

I would like to take this opportunity to thank my previous and current mentors for the opportunities to learn from them as well as my students and postdocs for the inspiring time working together. The Swiss National Science Foundation is gratefully acknowledged for funding part of this research under the SNSF Professorship Grant PP00P2_157615. The National Centre of Competence in Research (NCCR) MARVEL also supports part of the research under an Agility Plus Grant. Computational resources for our current work are provided by Ubelix, (http://www.id.unibe.ch/hpc), the HPC cluster at the University of Bern as well as the Swiss National Supercomputing Centre (CSCS) under project s766.

Received: February 25, 2018 


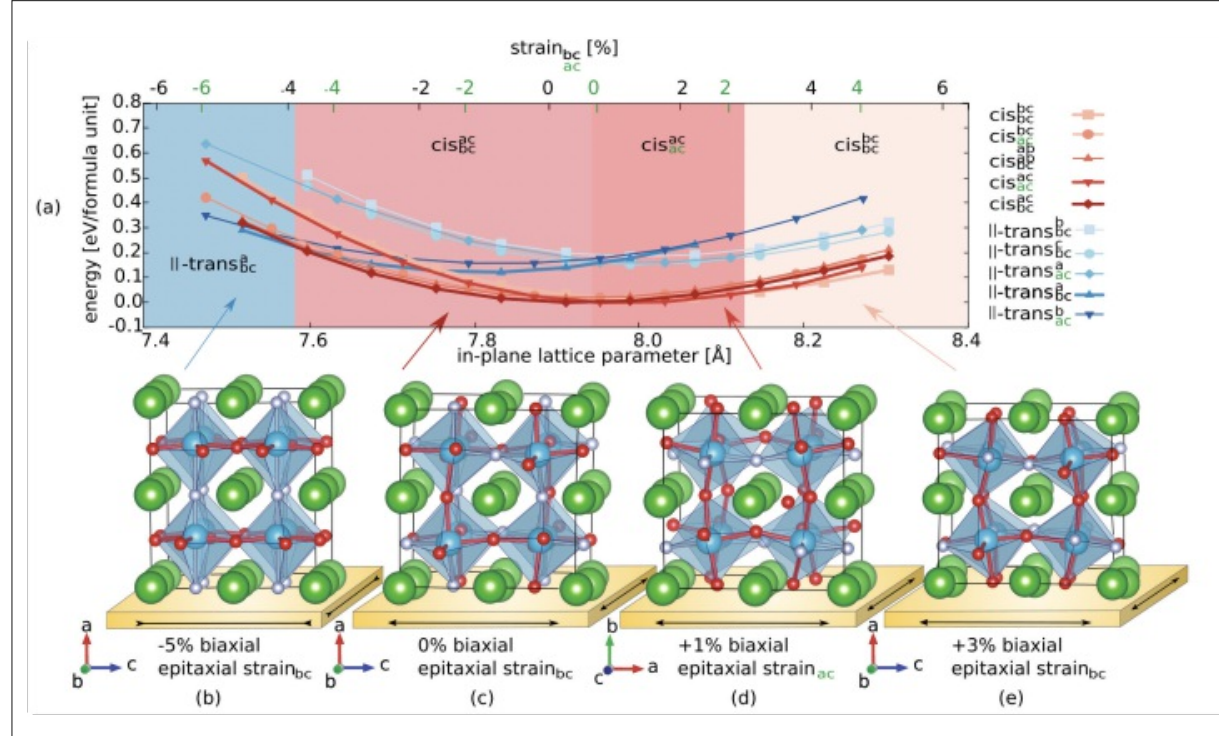

Fig. 7. Strain-dependent anion order in $\mathrm{LaTiO}_{2} \mathrm{~N}$ thin films. For compressive strain larger than $4 \%$ the material adopts a trans order and becomes ferroelectric. Reprinted with permission from ref. [33]. Copyright (2018), American Physical Society.

[1] J. D. Smith, W. G. Fahrenholtz, in 'Ceramic and Glass Materials: Structure, Properties and Processing', Eds. J. F. Shackelford, R. H. Doremus, Springer US, Boston, MA, 2008, p. 87.

[2] M. Batzill, Energ. Environ. Sci. 2011, 4, 3275.

[3] D. Kumar, R. Aluguri, U. Chand, T. Y. Tseng, Ceram. Int. 2017, 43, S547.

[4] S. Schweiger, R. Pfenninger, W. J. Bowman, U. Aschauer, J. L. M. Rupp, Adv. Mater. 2017, 29 , 1605049.

[5] J. M. Edge, Y. Kedem, U. Aschauer, N. A. Spaldin, A. V. Balatsky, Phys. Rev. Lett. 2015, 115, 247002.

[6] P. Hohenberg, W. Kohn, Phys. Rev. B 1964, 136, B864.

[7] W. Kohn, L. J. Sham, Phys. Rev. 1965, 140, 1133.

[8] J. Heyd, G. E. Scuseria, M. Ernzerhof, J. Chem. Phys. 2003, 118, 8207.

[9] J. Heyd, G. E. Scuseria, M. Ernzerhof, J. Chem. Phys. 2006, 124, 219906.
Langenberg, M. Trassin, J. Blasco, I. P. Krug, P. A. Algarabel, N. A. Spaldin, J. A. Pardo, M. Fiebig, Nat. Nanotechnol. 2015, 10, 661.

[18] R. Schaub, P. Thostrup, N. Lopez, E. Lægsgaard, I. Stensgaard, J. K. Nørskov, F. Besenbacher, 2001, 87, 266104.

[19] H. Cheng, A. Selloni, Phys. Rev. B 2009, 79, 092101.

[20] H. Cheng, A. Selloni, J. Chem. Phys. 2009, 131, 054703.

[21] M. Setvin, U. Aschauer, P. Scheiber, Y. F. Li, W. Hou, M. Schmid, A. Selloni, U. Diebold, Science 2013, 341, 988.

[22] M. Setvin, U. Aschauer, J. Hulva, T. Simschitz, B. Daniel, M. Schmid, A. Selloni, U. Diebold, J. Am. Chem. Soc. 2016, 138, 9565.

[23] A. Valdés, Z. W. Qu, G. J. Kroes, J. Rossmeisl, J. K. Nørskov, J. Phys. Chem. C 2008, 112, 9872.

[24] Y.-F. Li, Z.-P. Liu, L. Liu, W. Gao, J. Am. Chem. Soc. 2010, 132, 13008.

[25] S. Balaz, S. H. Porter, P. M. Woodward, L. J. Brillson, Chem. Mater. 2013, 25, 3337.

[26] S. G. Ebbinghaus, H.-P. Abicht, R. Dronskowski, T. Müller, A. Reller, A. Weidenkaff, Prog. Solid State Chem. 2009, 37, 173.

[27] J. P. Attfield, Crystal Growth \& Design 2013, $13,4623$.

[28] M. Yang, J. Oró-Solé, J. A. Rodgers, A. B. Jorge, A. Fuertes, J. P. Attfield, Nat. Chem. 2010, 3, 47.

[29] S. Ninova, U. Aschauer, J. Mater. Chem. A 2017, 5, 11040.

[30] M. Pichler, W. Si, F. Haydous, H. Téllez, J. Druce, E. Fabbri, M. E. Kazzi, M. Döbeli, S. Ninova, U. Aschauer, A. Wokaun, D. Pergolesi, T. Lippert, Adv. Funct. Mater. 2017, 27, 1605690.

[31] H. Ouhbi, U. Aschauer, in Preparation.

[32] M. Bouri, U. Aschauer, Phys. Chem. Chem. Phys. 2018, 20, 2771.

[33] N. Vonrüti, U. Aschauer, Phys. Rev. Lett. 2018, 120, 046001.

[34] A. Grimaud, O. Diaz-Morales, B. Han, W. T. Hong, Y.-L. Lee, L. Giordano, K. A. Stoerzinger, M. T. M. Koper, Y. Shao-Horn, Nat. Chem. 2017, 9, 457. 